For Computer Physics Communications: Proceedings of CCP 2004

\title{
Fluid Coexistence close to Criticality: Scaling Algorithms for Precise Simulation
}

\author{
Young C. Kim and Michael E. Fisher* \\ Institute for Physical Science and Technology, University of Maryland, College Park, Maryland 20742 USA
}

\begin{abstract}
A novel algorithm is presented that yields precise estimates of coexisting liquid and gas densities, $\rho^{ \pm}(T)$, from grand canonical Monte Carlo simulations of model fluids near criticality. The algorithm utilizes data for the isothermal minima of the moment ratio $Q_{L}\left(T ;\langle\rho\rangle_{L}\right) \equiv\left\langle m^{2}\right\rangle_{L}^{2} /\left\langle m^{4}\right\rangle_{L}$ in $L \times \cdots \times L$ boxes, where $m=\rho-\langle\rho\rangle_{L}$. When $L \rightarrow \infty$ the minima, $Q_{\mathrm{m}}^{ \pm}(T ; L)$, tend to zero while their locations, $\rho_{\mathrm{m}}^{ \pm}(T ; L)$, approach $\rho^{+}(T)$ and $\rho^{-}(T)$. Finite-size scaling relates the ratio $\mathbf{y}=\left(\rho_{\mathrm{m}}^{+}-\rho_{\mathrm{m}}^{-}\right) / \Delta \rho_{\infty}(T)$ universally to $\frac{1}{2}\left(Q_{\mathrm{m}}^{+}+Q_{\mathrm{m}}^{-}\right)$, where $\Delta \rho_{\infty}=\rho^{+}(T)-\rho^{-}(T)$ is the desired width of the coexistence curve. Utilizing the exact limiting $(L \rightarrow \infty)$ form, the corresponding scaling function can be generated in recursive steps by fitting overlapping data for three or more box sizes, $L_{1}, L_{2}, \cdots, L_{n}$. Starting at a $T_{0}$ sufficiently far below $T_{\mathrm{c}}$ and suitably choosing intervals $\Delta T_{j}=T_{j+1}-T_{j}>0$ yields $\Delta \rho_{\infty}\left(T_{j}\right)$ and precisely locates $T_{\mathrm{c}}$.

The algorithm has been applied to simulation data for a hard-core square-well fluid and the restricted primitive model electrolyte for sizes up to $L / a=8-12$ (where $a$ is the hard-core diameter): the coexistence curves can be computed to a precision of $\pm 1-2 \%$ of $\rho_{\mathrm{c}}$ up to $\left|T-T_{\mathrm{c}}\right| / T_{\mathrm{c}}=10^{-4}$ and $10^{-3}$, respectively. Universality of the scaling functions and the exponent $\beta$ is verified and the $\left(T_{\mathrm{c}}, \rho_{\mathrm{c}}\right)$ estimates confirm previous values based on data from above $T_{\mathrm{c}}$. The algorithm extends directly to calculating the diameter, $\rho_{\text {diam }}(T) \equiv \frac{1}{2}\left(\rho^{+}+\rho^{-}\right)$, and can lead to estimates of the Yang-Yang ratio. Furthermore, a new, explicit approximant for the basic scaling function $y$ permits straightforward estimates of $\Delta \rho_{\infty}(T)$ from limited $Q$-data when Ising-type criticality may be assumed.
\end{abstract}

\section{Introduction}

In recent years computer simulation has been an important tool to understand the critical behavior of fluids [1]. Various programming algorithms and techniques have been developed to enhance calculations with large-scale computers. However, determining phase boundaries, critical points and the universality classes of complex fluids, such as electrolytes, polymers, colloids, etc., has been and still is a great challenge. Here we present in detail, together with

\footnotetext{
* Corresponding Author: xpectnil@ipst.umd.edu
}

a new, 'economical' extension, a powerful method developed recently [2] to estimate precisely coexisting liquid and gas densities, $\rho^{+}(T)$ and $\rho^{-}(T)$, very close to the critical temperature, $T_{\mathrm{c}}$. Precise values of $\rho^{ \pm}(T)$ can then provide critical parameters and reveal critical exponents, via

$$
\Delta \rho_{\infty}(T) \equiv \rho^{+}-\rho^{-} \approx B|t|^{\beta}, t \equiv\left(T-T_{\mathrm{c}}\right) / T_{\mathrm{c}} .
$$

To determine $\rho^{ \pm}(T)$ in simulations it has been customary to observe the grand canonical equilibrium distribution function, $\mathcal{P}_{L}(\rho ; T)$, of the density, $\rho \equiv$ $N / V$, at constant $T<T_{\mathrm{c}}$, where $N$ and $V \equiv L^{d}$ are the particle number and volume of the system, 
respectively. For large $L$ below $T_{\mathrm{c}}$, the distribution $\mathcal{P}_{L}(\rho ; T)$ exhibits two well separated peaks located near $\rho^{ \pm}(T)$. Examining these peaks with aid of the equal-weight prescription [3] provides reasonable estimates for $\rho^{ \pm}(T)$. However, when $T$ approaches $T_{\mathfrak{c}}$, the peaks broaden, overlap strongly, and can no longer be separated uniquely thereby precluding reliable estimation of $\rho^{ \pm}(T)$ [4]. As well known, the underlying reason is the divergence of the bulk correlation length at criticality as $|t|^{-\nu}$.

To obtain better estimates of $\rho^{ \pm}(T)$ valid closer to $T_{\mathrm{c}}$, we examine instead the $Q$-parameter defined by [5]

$Q_{L}\left(T ;\langle\rho\rangle_{L}\right) \equiv\left\langle m^{2}\right\rangle_{L}^{2} /\left\langle m^{4}\right\rangle_{L}, \quad m=\rho-\langle\rho\rangle_{L}$,

where $\langle\cdot\rangle_{L}$ denotes a finite-size grand canonical expectation value at fixed $T$ and chemical potential, $\mu$, in a cubic box of dimensions $L \times L \times \cdots \times L$ with periodic boundary conditions. Below $T_{\mathrm{c}}$ one finds that $Q_{L}\left(T ;\langle\rho\rangle_{L}\right)$ exhibits two minima, say $Q_{\mathrm{m}}^{ \pm}(T ; L)$, at densities $\rho_{\mathrm{m}}^{ \pm}(T ; L)$ near $\rho^{ \pm}(T)$ [6]: see Fig. 1 . When

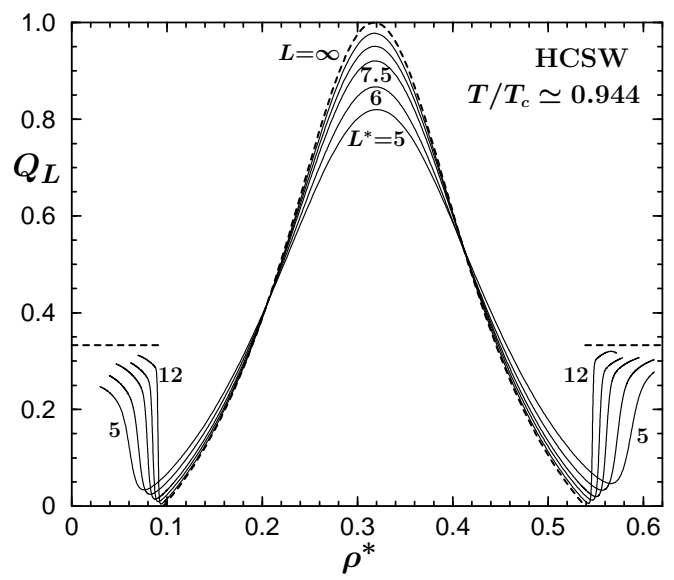

Fig. 1. Plots of simulation data for $Q_{L}\left(T ;\langle\rho\rangle_{L}\right)$ vs. $\rho^{*} \equiv\langle\rho\rangle a^{3}$ for the HCSW fluid at $T / T_{\mathrm{C}} \simeq 0.944$ showing minima that approach the limiting coexistence values $\rho^{+}(T)$ and $\rho^{-}(T)$. The solid curves are for $L^{*} \equiv L / a=12,9,7.5,6$ and 5 (where $a$ is the hard-core diameter); the dashed lines represent the exact limiting form (for the estimated values of $\rho^{+}$and $\rho^{-}$).

$L \rightarrow \infty$ the heights, $Q_{\mathrm{m}}^{ \pm}(T ; L)$, of these minima decay to zero while their locations, $\rho_{\mathrm{m}}^{ \pm}(T ; L)$, approach the desired coexistence values $\rho^{ \pm}(T)$. Thus understanding the behavior of the minima is potentially rewarding.

To that end, this article explains an unbiased scaling algorithm which utilizes calculated values of $Q_{\mathrm{m}}^{ \pm}(T ; L)$ and $\rho_{\mathrm{m}}^{ \pm}(T ; L)$ to obtain precise estimates of the coexistence-curve width or density discontinuity, $\Delta \rho_{\infty}(T)$. By "unbiased" we specifically mean that not only are the critical parameters $T_{\mathrm{c}}$ and $\rho_{\mathrm{c}}$ left open but, also, no assumptions regarding the value of the exponent $\beta$ in (1) or regarding the universality class of the critical point are made (in contrast to earlier approaches $[7,8])$. The algorithm has been applied to a hard-core square-well (HCSW) fluid and to the restricted primitive model (RPM) electrolyte. Although not demonstrated here, the algorithm extends directly $[2,9]$ to accurately estimate the diameter,

$\rho_{\text {diam }}(T) \equiv \frac{1}{2}\left[\rho^{+}(T)+\rho^{-}(T)\right]$.

Furthermore, studying $\left(Q_{\mathrm{m}}^{+}-Q_{\mathrm{m}}^{-}\right)$provides an effective way $[2,9]$ of estimating the strength, $\mathcal{R}_{\mu}$, of the Yang-Yang anomaly, namely, the relative divergence at criticality of the second derivative of the chemical potential on the phase boundary, $d^{2} \mu_{\sigma} / d T^{2}$ [10].

Finally, on the basis of an explicit expression, approximate but accurate, for a crucial scaling function relating $\Delta \rho_{\infty}(T)$ to the difference $\rho_{\mathrm{m}}^{+}(T)-\rho_{\mathrm{m}}^{-}(T)$, we demonstrate a simple, albeit biased algorithm that requires only limited data for the $Q$-minima: this should be valuable when, as usual, it may be safely assumed that the criticality is of Ising character $[7,8]$.

\section{Theoretical Background}

For sufficiently large $L$ at fixed $T<T_{\mathrm{c}}$ it is well established $[3,6]$ that the density distribution, $\mathcal{P}_{L}(\rho ; T)$, asymptotically approaches a sum of two Gaussian peaks which can be written as

$$
\begin{aligned}
\mathcal{P}_{L}(\rho ; T) \approx C_{L} & \left\{\chi_{-}^{-1 / 2} \exp \left[-\beta\left(\rho-\rho^{-}\right)^{2} L^{d} / 2 \chi_{-}\right]\right. \\
& \left.+\chi_{+}^{-1 / 2} \exp \left[-\beta\left(\rho-\rho^{+}\right)^{2} L^{d} / 2 \chi_{+}\right]\right\} \\
& \times \exp \left[\beta \rho\left(\mu-\mu_{\sigma}\right) L^{d}\right],
\end{aligned}
$$

where $\beta=1 / k_{\mathrm{B}} T$ while $\chi_{ \pm}(T)$ are the infinitevolume susceptibilities [defined via $\chi=(\partial \rho / \partial \mu)_{T}$ ] evaluated at $\rho=\rho^{ \pm}(T) \pm$, and $C_{L}(\mu, T)$ is a normalization factor. From this expression the parameter $Q_{L}\left(T ;\langle\rho\rangle_{L}\right)$ can be calculated readily and, in particular, the limiting form $Q_{\infty}\left(T ;\langle\rho\rangle_{\infty}\right)$, shown by the dashed lines in Fig. 1, can be derived. However, when criticality is approached at fixed $L$, the two-Gaussian 
representation of $\mathcal{P}_{L}(\rho ; T)$ becomes less accurate and it fails badly near criticality.

In the critical region, on the other hand, the behavior of $Q_{L}\left(T ;\langle\rho\rangle_{L}\right)$ can be understood via finite-size scaling theory[11], recently extended to incorporate pressure-mixing in the scaling fields $\tilde{t}$ and $\tilde{\mu}$ [12] (which is essential for describing the Yang-Yang anomaly [10]). For the $Q$-parameter, which depends on the three variables $L, T$, and $\langle\rho\rangle_{L}$, finite-size scaling provides the asymptotic, $t \rightarrow 0$, reduced, twovariable representation

$Q_{L}\left(T ;\langle\rho\rangle_{L}\right) \approx \mathbf{Q}\left(t L^{1 / \nu} ; \Delta \rho /|t|^{\beta}\right)$,

where $\mathcal{Q}(x, y)$ is the scaling function, while $\Delta \rho=$ $\langle\rho\rangle_{L}-\rho_{\mathrm{c}}$ and, as above, $\nu$ is the correlation length exponent.

It then follows that the minima, $Q_{\mathrm{m}}^{+}(T ; L)$ and $Q_{\mathrm{m}}^{-}(T ; L)$, and their corresponding displacements, $\left[\rho_{\mathrm{m}}^{+}(T ; L)-\rho_{\mathrm{c}}\right]$ and $\left[\rho_{\mathrm{c}}-\rho_{\mathrm{m}}^{-}(T ; L)\right]$, should, on approach to criticality, all reduce to functions of the scaled variable $x=t L^{1 / \nu}$ alone. Accordingly, the average of $Q_{\mathrm{m}}^{+}$and $Q_{\mathrm{m}}^{-}$and, using (1) and (3), the normalized density deviation ${ }^{1}$

$y \equiv\left[\langle\rho\rangle_{L}-\rho_{\text {diam }}(T)\right] / \Delta \rho_{\infty}(T)$,

should scale likewise. Thus we may anticipate (but should plan to check in applications) the scaling expressions

$$
\begin{aligned}
\bar{Q}_{\min }(T ; L) & \equiv \frac{1}{2}\left(Q_{\mathrm{m}}^{+}+Q_{\mathrm{m}}^{-}\right) \approx \mathcal{M}\left(t L^{1 / \nu}\right), \\
\Delta y_{\min }(T ; L) & \equiv\left(y_{\mathrm{m}}^{+}-y_{\mathrm{m}}^{-}\right)=\frac{\rho_{\mathrm{m}}^{+}-\rho_{\mathrm{m}}^{-}}{\Delta \rho_{\infty}(T)} \\
& \approx \mathcal{N}\left(t L^{1 / \nu}\right)
\end{aligned}
$$

where $\mathcal{M}(\cdot)$ and $\mathcal{N}(\cdot)$ are appropriate scaling functions, which when properly normalized should be universal.

Before proceeding further, notice that the normalizing divisor $\Delta \rho_{\infty}(T)$ in (6) and (8) is just the true width of the coexistence curve that we wish to estimate!

Now, at least in principle, one may eliminate the scaling variable $x=t L^{1 / \nu}$ between (7) and (8), e.g., by solving (7) for $x$ and substituting in (8), to obtain $\Delta y_{\text {min }}$ as a universal function of $\bar{Q}_{\text {min }}$, say, $\mathbf{y}(q)$. Of

\footnotetext{
1 The definition of $y$ adopted here differs from that used in [2] by a factor $\frac{1}{2}$.
}

course, this function is not known a priori. However, the two-Gaussian limiting form (4) for the density distribution $\mathcal{P}_{L}(\rho ; T)$, which is easily seen to obey scaling close to $T_{\mathrm{c}}$ when $x$ is large, can be used straightforwardly [12] to study the minima of $Q_{L}\left(T ;\langle\rho\rangle_{L}\right)$. In this limit we thence obtain the exact and universal expansion

$\Delta y_{\min }=1+\frac{1}{2} q+O\left(q^{2}\right)$,

in terms of the auxiliary variable

$q \equiv \bar{Q}_{\min } \ln \left(4 / e \bar{Q}_{\min }\right)$.

As we will explain, this result provides a route to the recursive, numerical construction of the full universal scaling function $\mathbf{y}(q)$ and, furthermore, to the evaluation of $\Delta \rho_{\infty}(T)$ and $T_{\mathrm{c}}$.

\section{Scaling Algorithm}

The basic idea of our algorithm is to fit data for the $Q$-minima to the formula (9), starting at some temperature $T_{0}$ far enough below $T_{\mathrm{c}}$ that the two-Gaussian form (4) is a good approximation, and then to extend the fits progressively to higher temperatures checking consistency with scaling, i.e., the uniqueness of $\boldsymbol{y}(q)$, as the calculations proceed. To make the fits, it is just the sought-for values of $\Delta \rho_{\infty}(T)$ that must be selected: and in order to vary $q$ in (9) and check the scaling, it is crucial to obtain simulation data for three or more fixed box sizes, say $L_{i}=L_{1}, L_{2}, \cdots, L_{n}(n \geq$ $3)$, at the same temperatures $T_{j}(j=0,1,2, \cdots)$.

It must also be stressed that high quality, precise data are essential. These can be obtained, as previous studies of the HCSW fluid [4] and the RPM [13] demonstrate, by careful simulation and the use of multiple histogram reweighting [14].

More formally, the initial step is to collect grand canonical Monte Carlo data sets for the $Q$-minima, $\left\{Q_{\mathrm{m}}^{ \pm}(T, L), \rho_{\mathrm{m}}^{ \pm}(T, L)\right\}$, generated at a sufficiently low $T_{0}$ as is to be verified by the ease of fitting to (9). This is illustrated in Fig. 2(a) using data for the HCSW fluid; but note, in particular, that the magnitude of the (positive) exponent $\psi$ is arbitrary and may be assigned any graphically convenient value (such as, e.g., $\psi=2$ or 5: see [2]). However, the reason for the choice made will be explained below. 


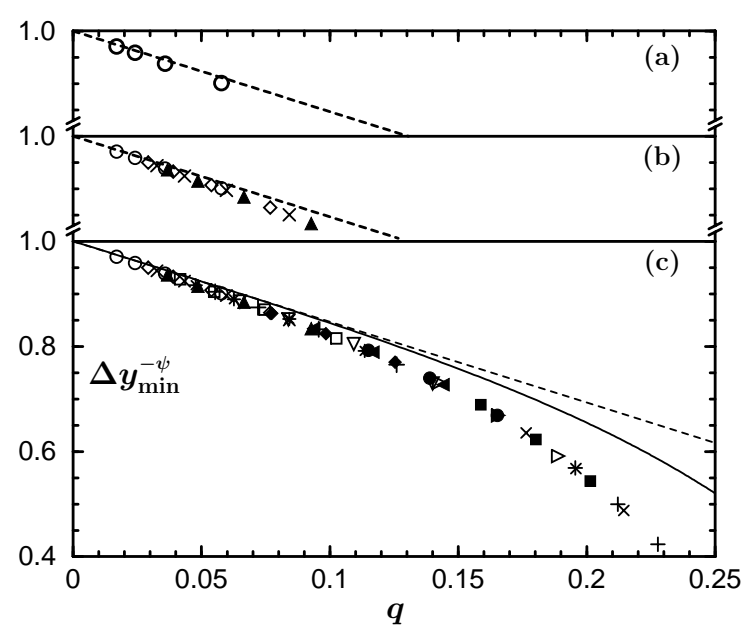

Fig. 2. Scaling plot of $\Delta y_{\min }^{-\psi}$ vs. $q \equiv \bar{Q}_{\min } \ln \left(4 / e \bar{Q}_{\min }\right)$ with $1 / \psi=0.326$ for the HCSW fluid; (a) at $T_{0} \simeq 0.903 T_{\mathrm{C}}$, (b) at $T_{0}$ and three higher temperatures up to $T \simeq 0.952 T_{\mathrm{c}}$, and (c) up to $T \simeq 0.985 T_{\mathrm{c}}$. The dashed lines represent the exact two-Gaussian limiting form to linear order in $q$; the solid curve in (c) portrays the full two-Gaussian approximation which evidently deviates significantly from the HCSW results even for $q \simeq 0.05$.

To generate the scaling function successfully, $n=$ 3 distinct box sizes may well suffice although $n=$ 4 has been used in our calculations. Furthermore, in order to avoid effects arising from corrections to the leading scaling behavior, the $L_{i}^{*} \equiv L_{i} / a$ should not be too small (where $a$ measures the particle size). For the HCSW fluid $L^{*} \gtrsim 7$ sufficed.

At an appropriate $T_{0}$ the value of $\bar{Q}_{\min }$ will be small: for our choice of $T_{0}$ for the HCSW fluid we had $\bar{Q}_{\text {min }} \lesssim 0.03$; but a somewhat larger value might provide acceptable accuracy. Following the prescription, one density-discontinuity value, say $\Delta \rho_{T_{0}}$ is then chosen for all the $L_{i}$ to provide the best fit of $\Delta y_{\min }^{(i)} \equiv\left[\rho_{\mathrm{m}}^{+}\left(T_{0}, L_{i}\right)-\rho_{\mathrm{m}}^{-}\left(T_{0}, L_{i}\right)\right] / \Delta \rho_{T_{0}}$ to the relation (9) with $q_{0}^{(i)} \equiv q\left(T_{0}, L_{i}\right)$. For the HCSW fluid this fit could be achieved accurately to within $\pm 0.2 \%$ of $\Delta \rho_{T_{0}}$. One may also check that the assignment of $\psi$ in any reasonable range has negligible effect on the fitting (which should be weighted more heavily on the smaller values of $\left.q_{0}^{(i)}\right)$. The optimal value $\Delta \rho_{T_{0}}$ is then identified as an estimate for $\Delta \rho_{\infty}\left(T_{0}\right)$.

The next step is to increase $T_{0}$ to $T_{1}=T_{0}+\Delta T_{0}$ and to compute the new data sets $\left\{\Delta y_{\min }\left(T_{1} ; L_{i}\right)\right\}_{i=1}^{n}$ and $\left\{q_{1}^{(i)} \equiv q\left(T_{1} ; L_{i}\right)\right\}_{i=1}^{n}$. In doing so, however, the crucial point is to select $\Delta T_{0}$ small enough that the new set $\left\{q_{1}^{(i)}\right\}_{i=1}^{n}$ overlaps the previous one $\left\{q_{0}^{(i)}\right\}_{i=1}^{n}$. When the new data set is in place, one must find, as before, a new value, $\Delta \rho_{T_{1}}$, such that the new data when plotted overlap and smoothly extend the previous data: see Fig. 2(b). The procedure thereby extends and numerically validates the scaling function up to larger values of $q$. The new value $\Delta \rho_{T_{1}}$ can then be taken as an optimal estimate for $\Delta \rho_{\infty}\left(T_{1}\right)$.

Subsequently, repeating these steps by increasing the temperature to $T_{j+1}=T_{j}+\Delta T_{j}$ will extend the scaling function further and generate successive estimates $\Delta \rho_{\infty}\left(T_{j}\right)$ for $j=2,3, \cdots$ : see Fig. 2(c). As $T_{\mathrm{c}}$ is approached, one will observe that smaller increments $\Delta T_{j}$ are needed and high quality data become increasingly important.

Since, via (1), $\Delta \rho_{\infty}(T) \rightarrow 0$ when $T \rightarrow T_{\mathrm{c}}-$ whereas the interval $\rho_{\mathrm{m}}^{+}(T ; L)-\rho_{\mathrm{m}}^{-}(T ; L)$ does not then vanish, the plot of $\Delta y_{\min }^{-\psi}(q)$ must approach zero at criticality. This behavior is clear in Fig. 3 which presents the full scaling function, $[\boldsymbol{y}(q)]^{-\psi}$, as con-

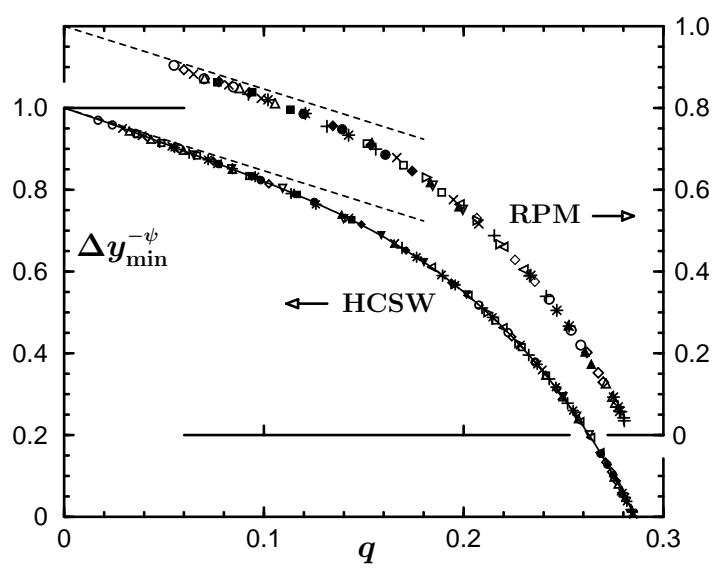

Fig. 3. Scaling plots of $\Delta y_{\min }^{-\psi}$ vs. $q$ for the HCSW fluid and the RPM with $1 / \psi$ identified with the Ising exponent $\beta \simeq 0.326$. The dashed lines again show the exact two-Gaussian limit to linear order while the solid curve (passing through the HCSW data) represents the approximant (12).

structed via the algorithm both for the HCSW fluid and for the RPM.

The vanishing of $\Delta y_{\min }^{-\psi}$ at $q=q_{\mathrm{c}} \simeq 0.2860$ generates unequivocal estimates for $T_{\mathrm{c}}$. For the HCSW fluid, a precision of \pm 2 parts in $10^{5}$ is realized: furthermore, the value for $T_{\mathrm{c}}$ agrees well with less pre- 
cise ( \pm 3 parts in $\left.10^{4}\right)$ estimates obtained by studying the model only above $T_{\mathrm{c}}$ [4].

The $Q_{L}\left(T ;\langle\rho\rangle_{L}\right)$ data for the RPM are harder to generate accurately and, moreover, as seen in Fig. 4, the variation of $Q_{L}$ with $\langle\rho\rangle_{L}$ turns out to be highly

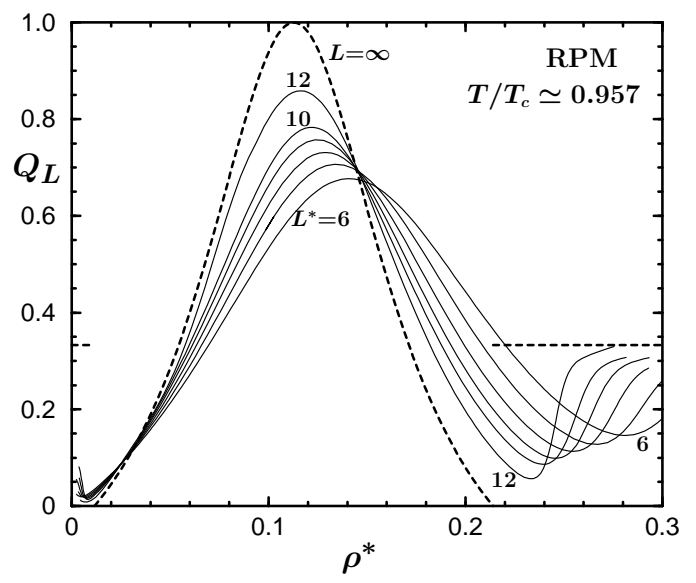

Fig. 4. Simulation data for the restricted primitive model (RPM) electrolyte as in Fig. 1; but notice the significantly greater asymmetry.

asymmetric, in strong contrast to the HCSW data in Fig. 1. (It might be remarked that the marked asymmetry of the RPM seems to be associated with a large, $\mathcal{R}_{\mu} \simeq 0.26$, value of the Yang-Yang ratio [2].) Nevertheless, the algorithm continues to work surprisingly well, yielding $\Delta \rho_{\infty}(T)$ to within $\pm 1-2 \%$ down to $|t| \simeq 10^{-3}$ and generating estimates for $T_{\mathrm{c}}$ with a precision of 4 parts in $10^{4}$ : these in turn agree completely with previous, $T>T_{\mathrm{c}}$ estimates [13]. It is important to note, furthermore, that to within the uncertainties, the RPM data in Fig. 3 fully confirm the expected universality of the scaling function $\boldsymbol{y}(q)$.

The analysis presented above demonstrates that $\Delta y_{\min }^{-\psi}$ must vanish linearly with $q$ if one sets $1 / \psi=$ $\beta$; but note again that this choice is not needed in order to estimate $T_{\mathrm{c}}$ reliably. However, the HCSW fluid is certainly expected to exhibit Ising-type criticality with $\beta_{\mathrm{Is}} \simeq 0.326$; and this is convincingly confirmed by the resulting plot of the coexistence curve shown in Fig. 5. Accordingly, $\psi=1 / \beta_{\text {Is }}$ was selected for use in Figs. 2 and 3. A bonus of the RPM calculations also displayed in Fig. 5 is that $\beta$ close to $\beta_{\text {Is }}$ again fits well. This result is of value since serious doubts have been raised regarding the universality class of ionic systems $[2,13,15,16]$.

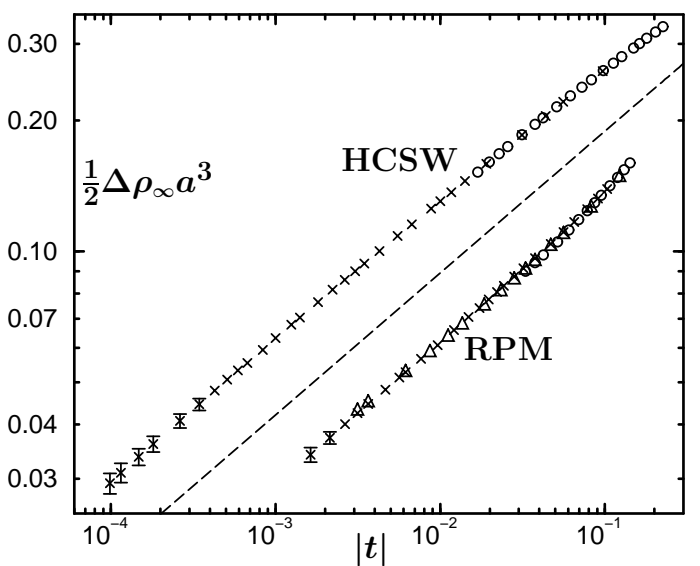

Fig. 5. A $\log -\log$ plot of the coexistence-curve half-width, $\frac{1}{2} \Delta \rho_{\infty} a^{3}$ vs. $t \equiv\left(T-T_{\mathrm{C}}\right) / T_{\mathrm{C}}$ (where $a$ is the hard-sphere diameter) for the HCSW fluid and for the RPM (at a $\zeta=5$ fine-discretization level $[2,13])$. The crosses follow from the full unbiased scaling algorithm while the circles for $|t|>10^{-2}$ report the best previous estimates (employing an equal-weight prescription). The triangles for the RPM are estimates obtained from the simple, economical (but biased) algorithm embodied in (11) and (12). The dashed line has a slope corresponding to $\beta=\beta_{\mathrm{Is}} \simeq 0.326$ : see (1).

\section{An Economical Biased Algorithm}

In practice, the full, unbiased scaling algorithm may be inconvenient for some applications since it requires a significant amount of precise data narrowly spaced in temperature, especially when $T_{\mathrm{c}}$ is approached. Furthermore, one needs to calculate reliably an initial set of $Q$ minima at sufficiently low $T$ that the twoGaussian structure of $\mathcal{P}_{L}(\rho ; T)$ is well obeyed. On the other hand, if one is prepared to accept that a model of interest exhibits Ising-type criticality, one can, in fact, utilize the HCSW scaling plot for $y_{\min }(T ; L)$ in Fig. 3 to estimate $\Delta \rho_{\infty}$ at any given $T$ !

To see this most clearly, recall that $\Delta y_{\min }(T ; L)$ is (for large enough $L$ and, say, $|t|=\left(T_{\mathrm{c}}-T\right) / T_{\mathrm{c}} \lesssim 0.1$ ) described by a universal scaling function, $\boldsymbol{y}(q)$, as Fig. 3 demonstrates. Then we may rewrite (8) in the direct form

$\Delta \rho_{\infty}(T) \approx\left[\rho_{\mathrm{m}}^{+}(T ; L)-\rho_{\mathrm{m}}^{-}(T ; L)\right] / \mathbf{y}[q(T ; L)]$,

where $q(T ; L)$ follows from (7) and (10). In words this simply says that $\mathbf{y}(q)$ acts as a correction factor that transforms the first approximation to the 
coexistence-curve width derived from the locations of the $Q$-minima, into the desired answer. Thus, for a selected temperature $T$ one need only locate the minima of $Q_{L}\left(T ;\langle\rho\rangle_{L}\right)$ (for a reasonable value of $L)$, determine the difference $\left(\rho_{\mathrm{m}}^{+}-\rho_{\mathrm{m}}^{-}\right)$, calculate $q$, and substitute in (11). As a wise precaution, using a second value of $L$ will enable one to check that corrections to scaling are negligible.

To facilitate this very simple, albeit biased procedure, we have fitted the HCSW data in Fig. 3 to an expression for $\boldsymbol{y}(q)$ that, with $\beta=\beta_{\text {Is }} \simeq 0.326$, embodies the linear vanishing when $q \rightarrow q_{\mathrm{c}} \simeq 0.2860$ and the exact small- $q$ behavior (9). Indeed, with $\tilde{q} \equiv$ $q / q_{\mathrm{c}}$, the approximant

$$
\begin{aligned}
& {[\boldsymbol{y}(q)]^{-1 / \beta}} \\
& \quad \simeq\left(1-\frac{q}{2 \beta}\right) \frac{(1-\tilde{q})\left(1+a_{2} \tilde{q}^{2}+a_{3} \tilde{q}^{3}\right)}{\left.1-\tilde{q}+b_{2} \tilde{q}^{2}+b_{3}\right] \tilde{q}^{3}},
\end{aligned}
$$

provides an excellent fit (see Fig. 3) for the coefficient values $a_{2}=1.829, a_{3}=1.955, b_{2}=2.340$, and $b_{3}=$ -1.388 .

We have tested this approach on the RPM (where Ising-type criticality is now well established $[13,16])$ : it yields the triangular data points shown in Fig. 5. These evidently agree well with the results of the full, unbiased algorithm. Thus we believe that the approximant (12) provides a convenient practical tool for accurately estimating the coexistence curves for a wide range of systems of Ising character.

\section{Summary}

In conclusion we have presented a novel scaling algorithm developed to estimate precisely the coexistence curves of asymmetric fluids near criticality. Both the fuller unbiased and a simpler biased approach have been illustrated using simulation data for the HCSW fluid and the RPM: precise and reliable estimates for $\Delta \rho_{\infty} \equiv \rho^{+}(T)-\rho^{-}(T)$ can be obtained even very close to $T_{\mathrm{c}}$. The biased approach, using (11) and the accurate scaling function representation (12), should prove especially useful in exploratory investigations. On the other hand, the full algorithm extends to yield estimates of the coexistence diameter, (3), and the Yang-Yang ratio $[2,10]$.

The support of the National Science Foundation (through Grant No. CHE 03-01101) is gratefully ac- knowledged. Jean-Noël Aqua and Sarvin Moghaddam kindly commented on a draft.

\section{References}

[1] See, e.g., A. Z. Panagiotopoulos, Mol. Simul. 9 (1992) 1.

[2] Y. C. Kim, M. E. Fisher, E. Luijten, Phys. Rev. Lett. 91 (2003) 065701.

[3] C. Borgs, S. Kappler, Phys. Lett. A 171 (1992) 37.

[4] G. Orkoulas, M. E. Fisher, A. Z. Panagiotopoulos, Phys. Rev. E 63 (2001) 051507.

[5] K. Binder, Z. Phys. B 43 (1981) 119.

[6] K. Binder, D. P. Landau, Phys. Rev. B 30 (1984) 1477; B. Dünweg, D. P. Landau, Phys. Rev. B 48 (1993) 14182.

[7] A. D. Bruce, N. Wilding, Phys. Rev. Lett. 68 (1992) 193.

[8] Y. C. Kim, M. E. Fisher, J. Phys. Chem. B 108 (2004) 6750.

[9] Y. C. Kim, M. E. Fisher, to be published.

[10] M. E. Fisher, G. Orkoulas, Phys. Rev. Lett. 85 (2000) 696.

[11] M. E. Fisher, M. N. Barber, Phys. Rev. Lett. 28 (1972) 1516.

[12] Y. C. Kim, M. E. Fisher, Phys. Rev. E 68 (2003) 041506.

[13] E. Luijten, M. E. Fisher, A. Z. Panagiotopoulos, Phys. Rev. Lett. 88 (2002) 185701.

[14] A. M. Ferrenberg, R. H. Swendsen, Phys. Rev. Lett. 63 (2002) 1195.

[15] H. Weingärtner, W. Schröer, Adv. Chem. Phys. 116 (2001) 1.

[16] Y. C. Kim, M. E. Fisher, Phys. Rev. Lett. 92 (2004) 185703. 